\title{
Efecto de la adición de copolímeros reactivos a mezclas de PLA/ATP
}

\section{Addition effect of reactive copolymers in PLA/TPS blends}

\author{
KANTUN-UICAB, Maria Cristinat**', RODRÍGUEZ-SÁNCHEZ, Isis”, RODRÍGUEZ-GONZÁLEZ, \\ Francisco Javier" ' y HORTELANO-CAPETILLO, Juan Gregorio'
}

'Universidad Politécnica de Juventino Rosas (UPJR), México.

"Instituto Politécnico Nacional, (UPIIG-IPN), México.

"'Centro de Investigación en Química Aplicada (CIQA), México.

ID $1^{\text {er }}$ Autor: Maria Cristina, Kantun-Uicab / ORC ID: 0000-0003-1588-5414, CVU CONACYT ID: 162342

ID $1^{\mathrm{er}}$ Coautor: Isis, Rodríguez-Sánchez / ORC ID: 0000-0002-6917-838X, CVU CONACYT ID: 103852

ID $2^{\text {do }}$ Coautor: Francisco Javier, Rodríguez-González / CVU CONACYT ID: 25352

ID $3^{\text {er }}$ Coautor: Juan Gregorio, Hortelano-Capetillo / ORC ID: 0000-0002-3702-4853, CVU CONACYT ID: 347496

DOI: $10.35429 /$ JOIE.2020.13.4.20.26

Recibido Enero 18, 2020; Aceptado Marzo 31, 2020

\begin{abstract}
Resumen
En este trabajo se estudió la compatibilización reactiva de mezclas de Acido Poliláctico/Almidón Termoplástico (PLA/ATP, 80/20\%w/w) utilizando copolímeros acrílicos de MMA-GMA. La compatibilización fue estudiada mediante reometría de torque, análisis térmico (DSC), Microscopía Electrónica de Barrido (MEB) y propiedades mecánicas. Durante el mezclado en fundido el torque de las mezclas PLA/ATP disminuyó, lo que se atribuyó al rompimiento de las cadenas de almidón. La adición del copolímero de MMA-GMA incrementó el torque durante el período de mezclado. Este incremento en el torque es una evidencia del incremento en la viscosidad atribuido a las reacciones entre los grupos epóxicos presentes en el copolímero acrílico, los grupos terminales del PLA y los grupos hidroxilo presentes en el ATP. Las morfologías de las mezclas compatibilizadas presentaron una disminución en el tamaño de partícula de los dominios del ATP y un incremento del $30 \%$ de elongación. La compatibilización reactiva es una técnica interesante para poder ampliar la versatilidad de materiales de PLA que puedan sustituir potencialmente a los materiales obtenidos a partir del petróleo.
\end{abstract}

Compatibilización reactiva, Ácido poliláctico, Almidón termoplástico

\begin{abstract}
In this work, the reactive compatibilization of Polylactic Acid/Thermoplastic Starch (PLA/TPS, 80/20\% w/w) blends using acrylic copolymers of methyl methacrylateglycidyl methacrylate (MMA-GMA) was examined. The compatibilization was studied using torque rheometry, thermal analysis (DSC), Scanning Electron Microscopy (SEM) and mechanical properties. During melt mixing, the torque of PLA/TPS blends decreased, this was related to the breakup of starch chains. The addition of MMAGMA copolymer increased the torque during the mixing period. This torque increasing is evidence of a viscosity increase and it was related to the reactions between the epoxy group present in the acrylic copolymer, the endgroups of the PLA and TPS hydroxyl groups. The morphologies of the compatibilized blends showed a decrease in the particle size of the TPS domains and an increase in elongation of $30 \%$. The reactive compatibilization is an interesting technique to expand the property range of PLA materials, which can potentially substitute oil-based materials.
\end{abstract}

Reactive compatibilization, Polylactic acid, Thermoplastic starch

Citación: KANTUN-UICAB, Maria Cristina, RODRÍGUEZ-SÁNCHEZ, Isis, RODRÍGUEZ-GONZÁLEZ, Francisco Javier y HORTELANO-CAPETILLO, Juan Gregorio. Efecto de la adición de copolímeros reactivos a mezclas de PLA/ATP. Revista de Ingeniería Innovativa. 2020. 4-13:20-26.

\footnotetext{
*Correspondencia al Autor (Correo Electrónico: mkantun_ptc@upjr.edu.mx)
}

$\dagger$ Investigador contribuyendo como primer autor. 


\section{Introducción}

El ácido poliláctico (PLA) es un polímero renovable que se obtiene a partir del ácido láctico (Auras, 2010). Tiene buenas propiedades de barrera y permeabilidad, alta resistencia y módulo de elasticidad. El PLA, es similar en varias propiedades al Polietiléntereftalato (PET). Este polímero es altamente transparente con una velocidad de cristalización relativamente baja, por lo que se vuelve candidato prometedor para la fabricación de películas orientadas biaxialmente, contenedores termoformados y botella soplada (Huneault, 2007).

Por otra parte, el almidón es un polímero natural y semicristalino que está conformado por dos tipos de polisacáridos, la amilosa y la amilopectina (Bertolini, 2009). La estructura cristalina del almidón desaparece cuando es sometido a temperaturas arriba de la gelatinización $\left(70-90{ }^{\circ} \mathrm{C}\right.$ ) en presencia de plastificantes (agua y glicerina) y esfuerzos de corte. De esta manera es como se obtiene el almidón termoplástico (ATP) y que dependiendo de la cantidad de plastificante se comporta desde un material rígido a uno dúctil (Utracki, 2002).

La técnica de compatibilización reactiva de mezclas poliméricas, consiste en la adición de un copolímero que debe ser miscible con uno de los componentes y reaccionar con un grupo funcional del otro componente con el fin de formar un copolímero in-situ (agente compatibilizante) durante el mezclado en fundido (Auras, 2010) (Cheremisinoff, 1997) (Liu N. B., 1992).

Cabe mencionar, que la eficiencia del agente compatibilizante generado, se evaluará midiendo: a) el grado de disminución de la tensión interfacial, b) si suprime la coalescencia de la fase dispersa y c) si aumenta la adhesión entre las fases (Auras, 2010) (Cheremisinoff, 1997) (Liu N. B., 1992). Sin embargo, todo lo anterior está en función del copolímero reactivo que se adiciona a la mezcla (Ali, 2017). Es decir, debe mantener un control en la cantidad de los grupos reactivos que van a generar el agente compatibilizante, el peso molecular podría afectar la difusión hacia la interfase y la concetración de este en la saturación de la interfase de la misma.
En este trabajo se presenta el estudio del efecto de la adición de copolímeros reactivos a mezclas de PLA/ATP para la generación de copolímeros in-situ (agente compatibilizante) durante el mezclado en fundido.

\section{Metodología}

Los materiales utilizados fueron PLA 2002D de Nature Works con un MFI de $9.9 \mathrm{~g} / 10 \mathrm{~min}$ (180 ${ }^{\circ} \mathrm{C}, 2.16 \mathrm{Kg}$ ). Almidón termoplástico con 29, 36 y $40 \%$ de glicerina en peso (ATP29, ATP36 y ATP40) obtenido de acuerdo al método reportado por (Tena, 2007).

El peso molecular promedio en número del homopolímero de MMA (HOMO) fue de Mn = 56547 Da y del copolímero de MMA-GMA (COPO) fue de $\mathrm{Mn}=55610 \mathrm{Da}$ y $4.7 \%$ en peso de grupos reactivos de GMA (dato obtenido de acuerdo a la norma ASTM D1652).

Las películas de PLA, el Homopolímero y el copolímero obtenidas por casting, se prepararon disolviéndolas en diclorometano. Estas fueron secadas durante 24 horas a temperatura ambiente y hasta peso constante en una estufa de convección con vacío continuo.

El PLA, las mezclas PLA/HOMO, PLA/COPO, PLA/ATP (80/20) y PLA/COPO/ATP $(77 / 3 / 20)$ fueron preparadas en una cámara de mezclado interno (Marca Brabender) a $180{ }^{\circ} \mathrm{C}$, durante 15 min a $60 \mathrm{RPM}$ y en atmósfera inerte. Enseguida se prepararon (con el material aún caliente), placas en una prensa con una presión de 20 ton. durante $8 \mathrm{~min}$ a $180^{\circ} \mathrm{C}$ para lograr un espesor de $1 \mathrm{~mm}$.

\section{Caracterízación de las mezclas de PLA/copolímero acrílico}

El estudio de la miscibilidad de las mezclas PLA/copolímero acrílico se evaluó por los cambios en la temperatura vítrea $\left(\mathrm{T}_{\mathrm{g}}\right)$ utilizando un calorímetro diferencial de barrido TA Instruments modelo MDSC2920, EUA. Las muestras se colocaron en cápsulas de aluminio calentándose a una velocidad de $10^{\circ} \mathrm{C} / \mathrm{min}$ de $0^{\circ}$ a $180{ }^{\circ} \mathrm{C}$, bajo atmósfera de Nitrógeno. 
Para la evaluación de las propiedades mecánicas de tracción de las mezclas, se utilizó una máquina de pruebas universales Instron (modelo 4301) de acuerdo a la norma ASTM D638. Las probetas utilizadas fueron tipo IV y fueron deformadas a $5 \mathrm{~mm} / \mathrm{min}$ con una celda de $50 \mathrm{KN}$.

Para el análisis morfológico, las muestras fueron revestidas con una aleación de oro/paladio y observadas en un microscopio electrónico de barrido (SEM) modelo JSM-820.

\section{Resultados}

En el Gráfico 1, se muestran los termogramas DSC del segundo calentamiento del PLA, el homopolímero de MMA y del copolímero reactivo MMA-GMA de las películas preparadas por casting. En los resultados se observan claramente las diferencias en las transiciones térmicas. En el caso del PLA, pueden observarse tres transiciones principales características de un polímero semicristalino. La temperatura de Transición Vítrea (Tg) fue detectada a los $59^{\circ} \mathrm{C}$, la Temperatura de Cristalización (Tc) a $124{ }^{\circ} \mathrm{C}$ y la Temperatura de fusión (Tm) a $166{ }^{\circ} \mathrm{C}$. En contraste, los polímeros acrílicos HOMO y COPO, presentaron una sola transición térmica, correspondiente a la $\mathrm{Tg}$ de 98 y $96{ }^{\circ} \mathrm{C}$ respectivamente. Este comportamiento térmico es característico de polímeros amorfos.

El Gráfico 2 muestra los termogramas DSC de las mezclas obtenidas en fundido. En el caso del PLA presentó transiciones similares a las obtenidas por casting con una $\mathrm{Tg}$ de $59^{\circ} \mathrm{C}$. Este resultado indica que el tratamiento térmico durante el procesamiento no afecta significativamente las características del PLA. Sin embargo, en el caso de las mezclas PLA/HOMO y PLA/COPO (80/20) después del procesamiento únicamente se detectó una transición. El efecto evidente fue en la cristalinidad. En la literatura se ha reportado que los polímeros acrílicos tienen ciertas interacciones con las zonas amorfas del PLA (Zhang, 2003) (Eguiburu, 1998). En el caso de la mezcla PLA/COPO, se obtiene un corrimiento de la Tg hacia la derecha. Este cambio puede deberse a que durante el mezclado en fundido el PLA puede aumentar las interacciones con el grupo epóxido del GMA (Liu H. S., 2011) (ver Figura 1).
Es decir, el producto de la reacción entre los grupos del extremo carboxilo o hidroxilo del PLA y los grupos epoxy del copolímero produciría menor libertad de movimiento molecular del PLA.

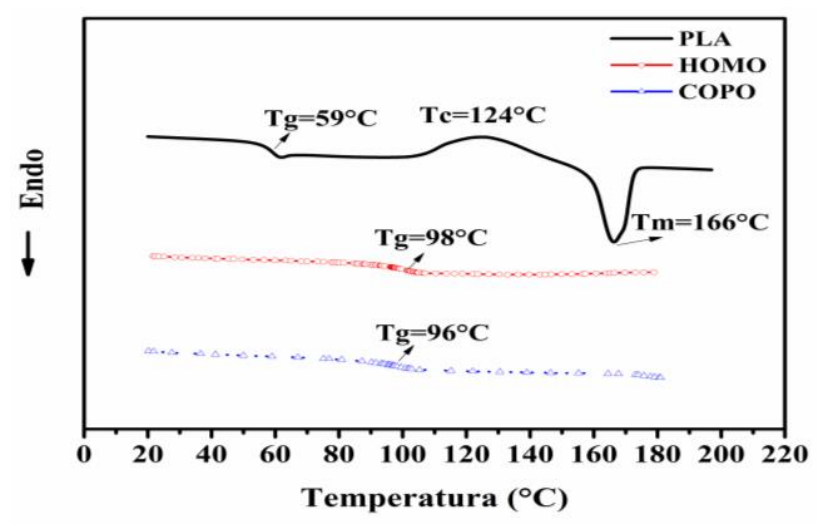

Gráfico 1 Termograma de DSC del PLA, del Homopolímero de MMA y del copolímero reactivo MMA-GMA

Fuente: elaboración propia [OriginLab]

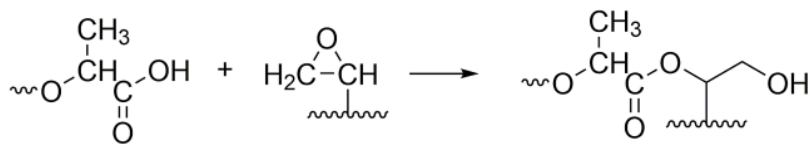

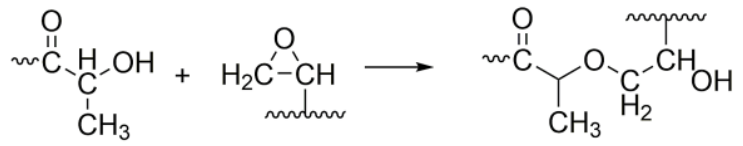

Figura 1 Posibles reacciones del grupo epóxido con los grupos terminales del PLA

Fuente: elaboración propia [ChemDraw]

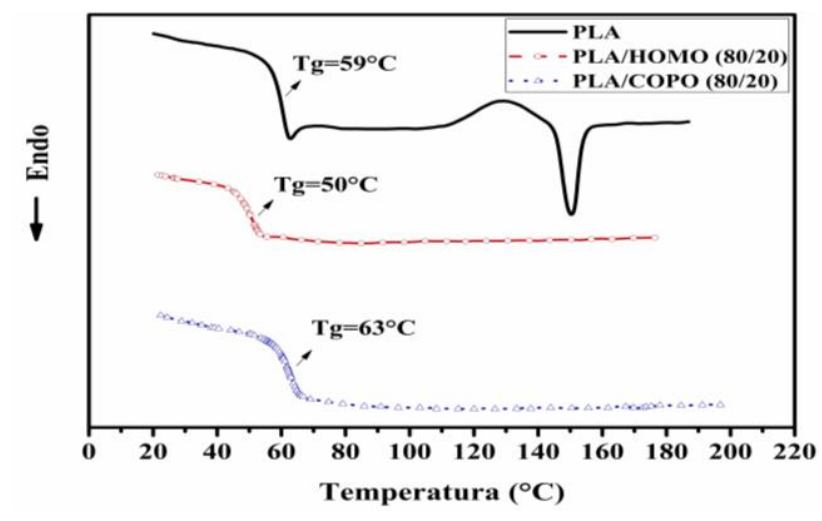

Gráfico 2 Termograma de DSC de las mezclas después del procesamiento del PLA/Homopolímero y del PLA/COPO (80/20)

Fuente: elaboración propia [OriginLab]

Con el fin de facilitar el análisis del efecto del torque producido por el mezclado, se decidió descartar los primeros 6 min. de procesamiento debido a la estabilización en el valor del torque. En el Gráfico 3 se muestran las mediciones del torque de las mezclas PLA/ATP's.

KANTUN-UICAB, Maria Cristina, RODRÍGUEZ-SÁNCHEZ, Isis, RODRÍGUEZ-GONZÁLEZ, Francisco Javier y HORTELANO-CAPETILLO, Juan Gregorio. Efecto de la adición de copolímeros reactivos a mezclas de PLA/ATP. Revista de Ingeniería Innovativa. 2020 
En el caso del PLA, el torque disminuye un $14.5 \%$ después de los 15 min de mezclado. Para las mezclas de PLA/ATP's se observa una reducción al aumentar la cantidad de glicerina en el ATP que podría atribuirse a que: la glicerina actua como un plastificante, existe la probable ruptura de cadenas de almidón por el procesamiento y las mezclas tienes poca estabilidad termomecánica. El abatimiento del torque fue más significativo en el caso de ATP40 que perdió el $66.6 \%$ del torque con respecto al PLA. Este comportamiento se ha reportado para sistemas de mezclas en las que los componentes son incompatibles (Rodriguez-Gonzalez, 2003).

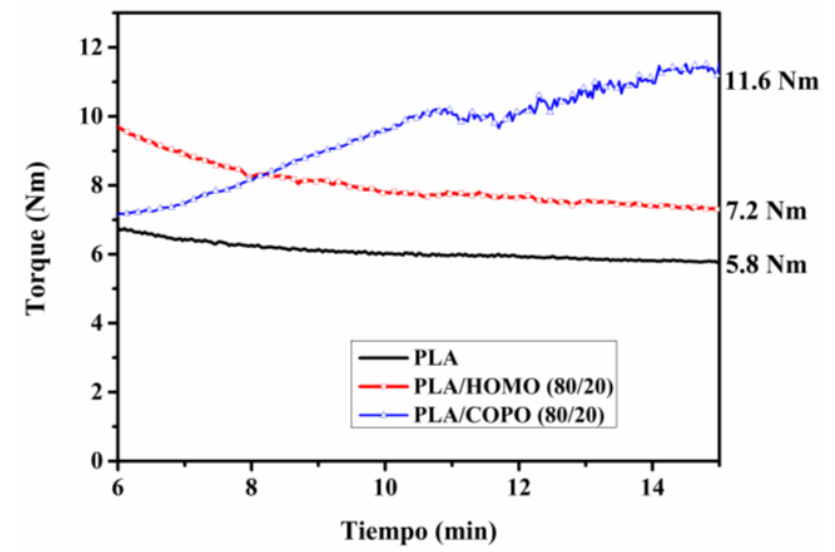

Gráfico 3 Reometría de torque de las mezclas de PLA/HOMO y del PLA/COPO (80/20)

Fuente: elaboración propia [OriginLab]

En el Gráfico 3, se muestra el efecto en el torque cuando la fase dispersa es el copolímero acrílico. La mezcla de PLA/HOMO aumentó a los 6 minutos a un valor de $9.8 \mathrm{Nm}$, sin embargo, en el transcurso del mezclado se observa una disminución paulatina del torque hasta un valor de 7.2 Nm. Este resultado comprueba que el copolímero acrílico no podría reaccionar durante el procesamiento con el PLA. En el caso de las mezclas PLA/COPO (80/20), a los 6 minutos se incrementó ligeramente con respecto al PLA, pero al terminar el tiempo de mezclado se oberva un incremento al doble del valor final del torque con respecto al PLA. Este efecto se podría atribuir a la existencia de reacciones entre los grupos terminales del PLA y el copolímero acrílico (Ver Figura 1). Estos mismos efectos, en el torque por la adición de un copolímero reactivo se han reportado en varios estudios (Willis, 1988) (Liu H. S., 2011). Esto, puede correlacionarse con los resultados obtenidos por DSC, en los que la $\mathrm{Tg}$ de esta mezcla aumentó ligeramente con respecto al PLA.

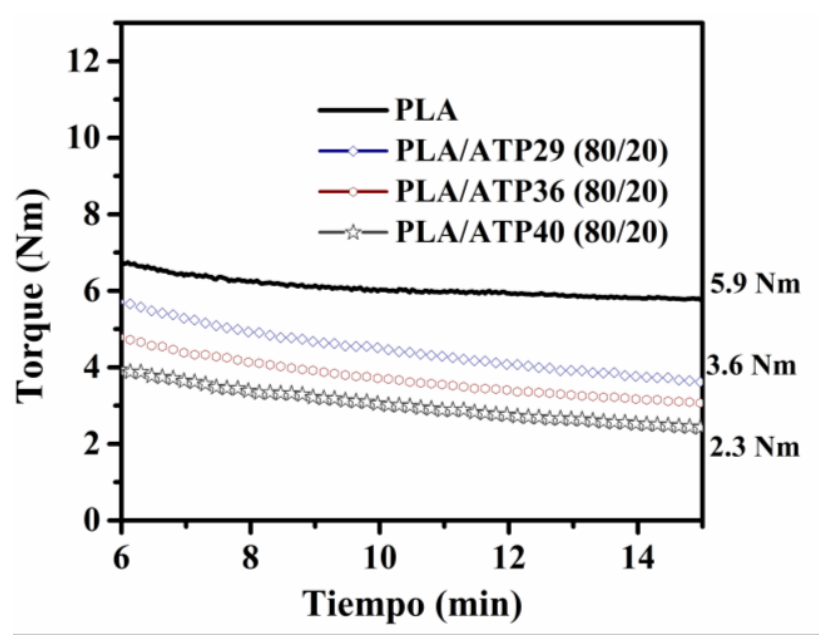

Gráfico 4 Reometría de torque de PLA y las mezclas de PLA/ATP (80/20) en función del contenido de glicerina en el ATP

Fuente: elaboración propia [OriginLab]

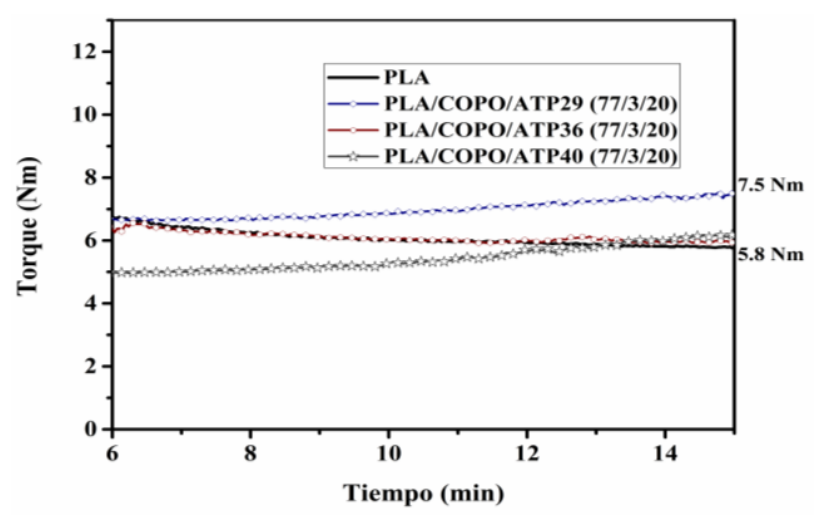

Gráfico 5 Reometría de torque de PLA y las mezclas de PLA/COPO/ATP (77/3/20) en función del contenido de glicerina en el ATP

Fuente: elaboración propia [OriginLab]

El Gráfico 5, muestra el efecto en el torque al adicionar 3\% del copolímero acrílico en función de la cantidad de glicerina en el ATP. Se observa que independientemente de la cantidad de glicerina el torque es mayor desde los 6 minutos en todas las mezclas con respecto a las que no tienen copolímero (ver Gráfico 4). El aumento en el torque es una prueba de la compatibilización reactiva durante el mezclado en fundido. En el caso de la mezcla de PLA/COPO/ATP29 se obtuvo un aumento en el torque de $1.7 \mathrm{Nm}$ al finalizar el mezclado. En este caso, el incremento estaría relacionado con las posibles reacciones con el copolímero y además a las propiedades intrínsecas del ATP29 (alta viscosidad).

En el caso de PLA/COPO/ATP40, el torque se elevó de $5 \mathrm{Nm}$ a los 6 minutos a un valor de $6.2 \mathrm{Nm}$ los 15 minutos rebasando ligeramente al PLA. 
Esto sugiere la existencia de interacciones específicas en la interfase de los dos componentes poliméricos, lo cual da indicios de que los grupos hidroxilo presentes en el ATP reaccionan con los grupos epoxi a tiempos cortos de mezclado para dar lugar al copolímero injertado in-situ (agente compatibilizante). Sin embargo, el torque sigue incrementándose durante todo el tiempo de mezclado, esto podría deberse a que el PLA también puede tener reacciones químicas con el copolímero acrílico (Liu H., 2011) (Li, 2011).
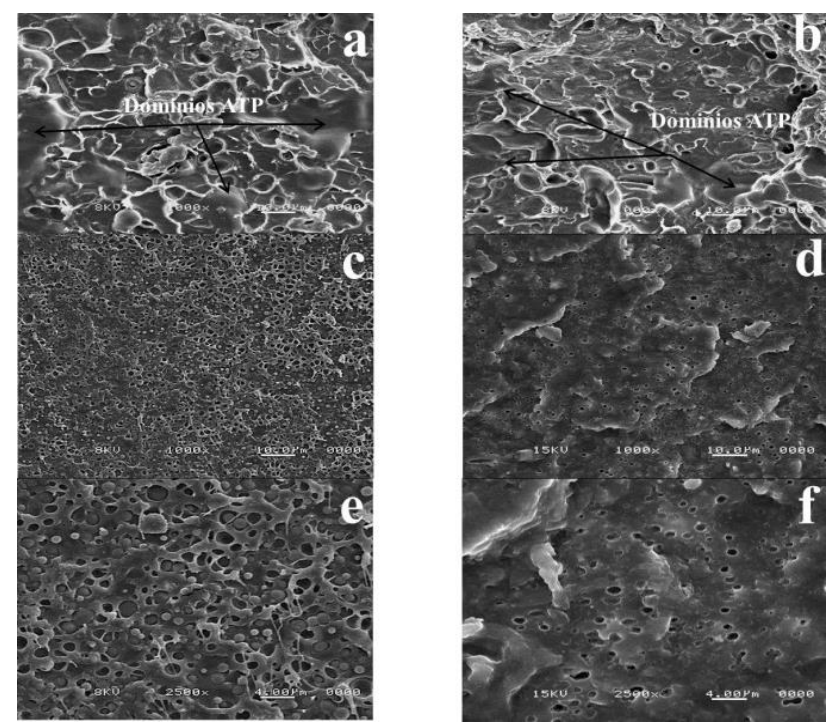

Figura 2 Micrografías de las mezclas de a) PLA/ATP36, b) PLA/ATP40 (80/20), c) y e) PLA/COPO/ATP36 y d) y f) PLA/COPO/ATP40 (77/3/20)

Fuente: elaboración propia [Microscopio electrónico de barrido (SEM) modelo JSM-820]

En la Figura 2 se pueden observar las morfologías de las mezclas PLA/ATP36, PLA/ATP40 (80/20), y el efecto de la adición del copolímero acrílico a las mezclas PLA/COPO/ATP36 y PLA/COPO/ATP40 (77/3/20). En la Figura 2a y en la Figura 2b, de las mezclas sin copolímeros acrílicos se pueden apreciar que los dominios de ATP36 y ATP40 en la mezcla con PLA respectivamente, son de formas irregulares es decir presentan una morfología gruesa y heterogénea. Además, no se nota evidencia de adhesión entre la fase ATP y la matriz de PLA. Es decir, las cavidades que se presentan en la matriz se deben principalmente al desprendimiento de partículas de la fase menor, las cuales estaban unidas sólo por fuerzas mecánicas, siendo por tanto la adherencia muy débil. En contraste, cuando se adicionó el 3\% de copolímero a la mezcla (Figura $2 \mathrm{c}-\mathrm{e}$ ), producen cambios muy notables en la morfología.
La dimensión de las partículas que forman la fase dispersa ha disminuido a tal grado que ahora la apariencia es homogénea. Estos cambios se deben a que hay una disminución de la tensión interfacial entre las fases, además es la confirmación de la formación del copolímero insitu. Estos resultados pueden correlacionarse con el aumento en el torque durante el mezclado en fundido. En la mezcla PLA/COPO/ATP36 (Figuras 2c y 2e) se observa un mayor número y tamaño de partículas dispersa del ATP con respecto a la mezcla PLA/COPO/ATP40 (Figura $2 \mathrm{~d}$ y 2f). Esta diferencia puede deberse al incremento en la cantidad de glicerina en el ATP (Taguet, 2009), ya que los grupos hidroxilo pueden reaccionar con los grupos epóxido del copolímero acrílico durante el mezclado y en consecuencia disminuir la cantidad de grupos reactivos reales con el ATP (Winthrop, 2014).

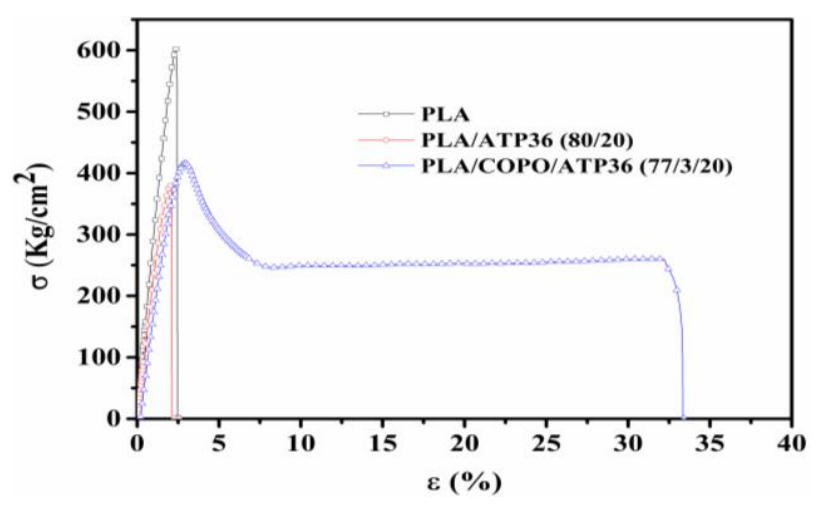

Gráfico 6 Propiedades mecánicas a la tensión del PLA, las mezclas de PLA/ATP36 (80/20) y PLA/COPO/ATP36 $(77 / 3 / 20)$

Fuente: elaboración propia [OriginLab]

Las curvas esfuerzo vs deformación del PLA, PLA/ATP36 (80/20) y de la mezcla PLA/COPO/ATP36 (77/3/20), se muestran en la Gráfico 6. En el caso de la mezcla PLA/ATP36, se observa que hay una disminución en la elongación y en la resistencia, pero el módulo de elasticidad disminuye ligeramente con respecto al PLA debido a la incompatibilidad de las fases. Sucede lo contrario con las mezclas con copolímero reactivo. En la mezcla PLA/COPO/ATP36 hay un aumento del 2.3\% hasta un $33 \%$ en la elongación con respecto a la mezcla de PLA/ATP36. También hay un aumento en la resistencia, esta es una medida indirecta de una mejora en la adhesión interfacial, lo que se traduce a una mejora en la transferencia de esfuerzos de la matriz a la fase dispersa (Akrami, 2016). 

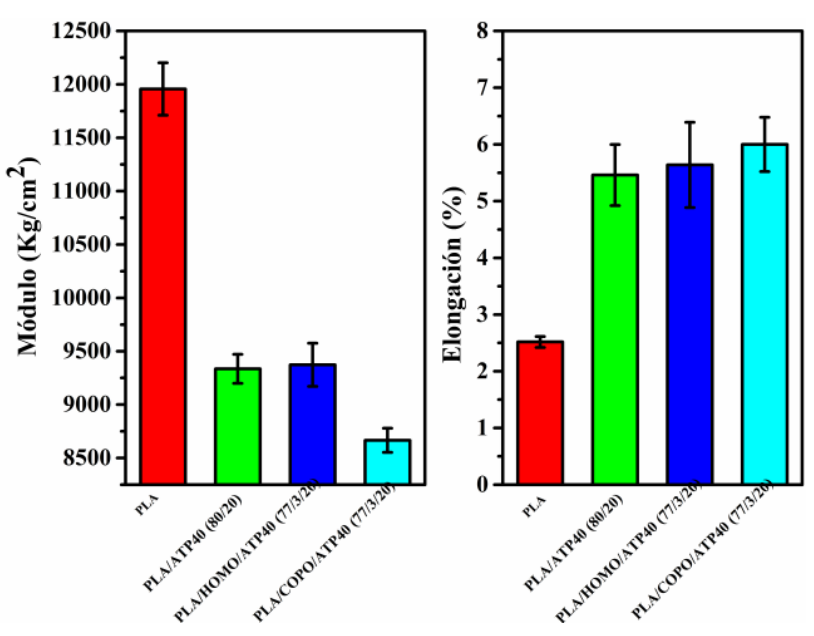

Gráfico 7 Propiedades mecánicas a la tensión del PLA, las mezclas de PLA/ATP40 (80/20) y PLA/COPO/ATP40 (77/3/20)

Fuente: elaboración propia [OriginLab]

Como era de esperarse, al incorporar al almidón termoplástico más dúctil al PLA, uno de los principales efectos es en el módulo de elasticidad (Gráfico 7). En el caso de la mezcla PLA/ATP40, disminuyó un $78 \%$ y la elongación se elevó un más del doble con respecto al PLA. Indicando la transición de una mezcla frágil a una dúctil. En la misma gráfica, se muestra el efecto en el módulo y la elongación de las mezclas con el homopolímero y el copolímero acrílico. Cuando no se tienen grupos reactivos en el copolímero, el módulo es similar a la mezcla de PLA/ATP40, sin embargo, cuando se incorpora 3\% del copolímero reactivo, hay una disminución del 72\% al compararlo con el PLA. Analizando la elongación se puede observar que hay un ligero incremento en la mezcla compatibilizada.

\section{Conclusiones}

En este trabajo se pudieron obtener mezclas de PLA/ATP compatibilizadas exitósamente con un copolímero de MMA-GMA. El análisis térmico de las mezclas de PLA y copolímeros acrílicos preparadas por mezclado en fundido mostró que estas son inmiscibles, sin embargo, el incremento en el torque durante el mezclado en fundido indica una reactividad importante entre los grupos terminales del PLA y el grupo epóxido del GMA.

El mezclado en fundido de las mezclas PLA/ATP puso en evidencia la inestabilidad térmica y mecánica de este sistema ya que el torque disminuyó durante todo el tiempo de mezclado.
Esta reducción en el torque de las mezclas puso en evidencia la inestabiliadd termomecánica del ATP, ya que el torque del PLA se mantuvo estable durante el proceso de mezclado.

La incorporación de un copolímero reactivo a las mezclas de PLA/ATP incrementó el torque durante el procesamiento, este efecto es debido principalmente a las reacciones entre los grupos hidroxilo del ATP y los grupos epóxido del GMA presente en el copolímero acrílico.

El análisis cualitativo de la morfología corroboró la disminución de la tensión interfacial ya que se observó una disminución del tamaño de partícula de la fasede ATP, de indicando la efectividad de la compatibilización reactiva. Sin embargo, también se observó que la cantidad de glicerina en el ATP afecta la dispersión de este en el PLA.

Las propiedades mecánicas de las mezclas compatibilizas con los copolímeros acrílicos presentaron una mejora en la elongación estando directamente relacionada con la cantidad de glicerina en el ATP.

\section{Referencias}

Akrami, M. (2016). A new approach in compatibilization of the poly (lactic acid)/thermoplastic starch (PLA/TPS) blends. Carbohydrate polymers , 144, 254-262.

Ali, S. (2017). From microstructure to mechanical properties of compatibilized polylactide/thermoplastic starch blends. Journal of Applied Polymer Science, 136 (16).

Auras, R. (2010). Poly(Lactic Acid): Synthesis, Structures, Properties, Processing, and Applications. Wiley.

\section{Bertolini, A. (2009). Starches: Characterization,} Properties, and Applications. CRC Press.

Cheremisinoff, P. (1997). Handbook of Engineering Polymeric Materials. CRC Press.

Eguiburu, J. (1998). Blends of amorphous and crystalline polylactides with poly (methyl methacrylate) and poly (methyl acrylate): a miscibility study. Polymer, 39 (26), 6891-6897. 
Huneault, M. (2007). Morphology and properties of compatibilized polylactide/thermoplastic starch blends. Polymer, 48.

Li, H. (2011). Effect of chain extension on the properties of PLA/TPS blends. Journal of Applied Polymer Science , 122 (1), 134-141.

Liu, H. (2011). Interaction of microstructure and interfacial adhesion on impact performance of polylactide (PLA) ternary blends. Macromolecules, 44 (6), 1513-1522.

Liu, N. B. (1992). Reactive polymers for blend compatibilization. Advances in Polymer Technology: Journal of the Polymer Processing Institute, 11 (4), 249-262.

Rodriguez-Gonzalez, F. (2003). High performance LDPE/thermoplastic starch blends: a sustainable alternative to pure polyethylene. Polymer, 44 (5), 1517-1526.

Taguet, A. (2009). Interface/morphology relationships in polymer blends with thermoplastic starch. Polymer, 50 (24), 57335743.

Tena, C. (2007). Estudio de la biodegradabilidad de mezclas de LDPE/Almidón termoplástico.

Utracki, L. (2002). Polymer blends handbook (Vol. 1). Kluwer academic publishers.

Willis, J. B. (1988). Processing-morphology relationships of compatibilized polyolefin/polyamide blends. Part I: The effect of an ionomer compatibilizer on blend morphology. Polymer Engineering and Science, 28 (21), 1416-1426.

Winthrop, C. (2014). New Developments in Polylactic Acid Research: Polymer Science and Technology Series. Nova Science Publishers.

Zhang, G. Z. (2003). Miscibility and phase structure of binary blends of polylactide and poly(methyl methacrylate). Journal of Polymer Science, Part B: Plymer Physics, 41 (1), 23-30. 